Department of Ophthalmology, Queen's Medical Centre, Nottingham, UK

2 Division of Primary Care, School of Medicine, University of Nottingham, UK

3 Department of Ophthalmology, Forth Valley NHS Trust, Stirling, UK

4 Academic Ophthalmology, Division of Clinical Neuroscience, School of Medicine, University of Nottingham, Nottingham, UK

Correspondence to: D S J Ting; ting.darren@gmail.com, darren.ting1@nottingham.ac.uk Cite this as: BMJ 2021;373:n1490 http://dx.doi.org/10.1136/bmj.n1490 Published: 25 June 2021

10-MINUTE CONSULTATION

\title{
Virtual consultation for red eye
}

\section{Charlotte Shan Ho, ${ }^{1}$ Anthony John Avery, ${ }^{2}$ lain A T Livingstone, ${ }^{3}$ Darren Shu Jeng Ting ${ }^{1,4}$}

\section{What you need to know}

- Virtual consultation, when performed in a systematic fashion, is a safe alternative to face-to-face examination to diagnose and manage patients with acute red eye(s)

- Advise the patient to report if symptoms remain unchanged or worsen, as important diagnoses may be missed at the initial virtual consultation

- Arrange prompt ophthalmology referral for patients with a red eye and symptoms such as ocular pain, ipsilateral headache, loss of vision or double vision, photophobia, history of trauma or surgery, corneal haziness, or systemic signs of infection

A 63 year old woman contacts her general practice, via a telephone call, reporting a left painful red eye since yesterday. Because of the covid-19 pandemic, she is booked in for a video consultation with a GP the same day.

Face-to-face consultations remain the optimal medium for ophthalmic consultation. Virtual consultations have been implemented as an alternative in the covid-19 pandemic. ${ }^{1-9}$ In a recent London study of 854 patients, video consultations for emergency ophthalmology services in adults had similar safety to face-to-face consultations. There was a higher rate of unplanned reattendance, but most patients were satisfied with video consultations. ${ }^{10}$

Red eye is a common ophthalmic presentation in primary care, accounting for 2 to $3 \%$ of the consultations. ${ }^{11}$ Virtual consultation for red eye(s) follow the same format and principles as face-to-face consultations. Performing ocular examination remotely can be challenging. Adaptation with specific instructions to the patient and/or family member will be required.

\section{What you should cover}

\section{Telephone consultation}

You may ask for history of presenting symptoms over a telephone consultation to determine the cause and severity of red eye. A telephone consultation also provides an opportunity to advise patients on any immediate measures that need to be taken while awaiting an appointment for a virtual video examination (such as not wearing contact lenses if conjunctival or corneal infection is suspected). Ask about red flag signs and symptoms (box 1), which should prompt ophthalmology referral.

Box 1: Red flag symptoms and signs for red eye Symptoms
- Ocular pain (acute onset, progressive nature, pain not relieved by analgesia or keeping the patient awake at night)

- Headache on the same side as the affected eye

- Visual loss

- Photophobia

- Recent history of contact lens wear, trauma, or ocular surgery

- New onset of binocular double vision

Signs

- Periorbital swelling or rashes respecting the midline of the face

- Proptosis

- Eyeball tenderness on palpation

- Corneal whitening (due to infection, severe inflammation, or oedema)

- Non-reactive pupil

- Systemic signs of infection (such as fever, chills, and being generally unwell)

\section{Virtual examination}

After a detailed history (box 2), a subsequent video consultation is desirable but may not always be necessary, for example, if a diagnosis can be made confidently (such as conjunctivitis) or if the patient warrants a face-to-face ophthalmic examination (such as corneal infection with severe painful red eye and visual loss).

\section{Box 2: Pointers for history taking in a patient with red} eye(s)

- Is it painful? Red eye with no pain or only mild discomfort is usually self limiting (fig 1). Painful red eye usually points towards more serious causes such as corneal infection, ${ }^{12}$ anterior uveitis, ${ }^{13}$ acute angle-closure glaucoma, ${ }^{14}$ or scleritis. ${ }^{15}$ In the presence of ocular pain, explore the onset, duration, progression, laterality, and severity of the pain. Ask about red flag symptoms that indicate serious conditions (box 1). Photophobia is suggestive of corneal pathologies or anterior uveitis. Pain on eye movements suggests orbital pathology.

- Is it unilateral or bilateral? Acute painful red eye conditions-including corneal infection, acute angle-closure glaucoma, and scleritis-often present unilaterally. These are potentially sight threatening. Anterior uveitis may present unilaterally or bilaterally. Unilateral conjunctivitis should be a diagnosis of exclusion, after ruling out any red flag symptoms and signs.

- Is there any discharge? Ocular discharge and itching are commonly associated with conjunctivitis. Viral 
conjunctivitis usually produces watery mucous discharge, whereas bacterial conjunctivitis produces mucopurulent discharge with crusting of the eyelashes. ${ }^{16}$

- Is the vision affected? Loss of vision in red eye suggests a more serious cause, particularly when the loss of vision is sudden, severe, or progressive. Reduced visual acuity with painful red eye, halos, and headache are indicative of acute angle-closure glaucoma. Serious red eye conditions such as anterior uveitis and anterior scleritis may not present with visual loss during the initial stage. New onset of double vision with red eye is suggestive of orbital pathologies, including orbital cellulitis and thyroid eye disease.

- Any contact lens wear? A painful red eye in contact lens wearers should raise the suspicion of contact lens-related corneal infection. This requires an urgent ophthalmic assessment within 24 hours.

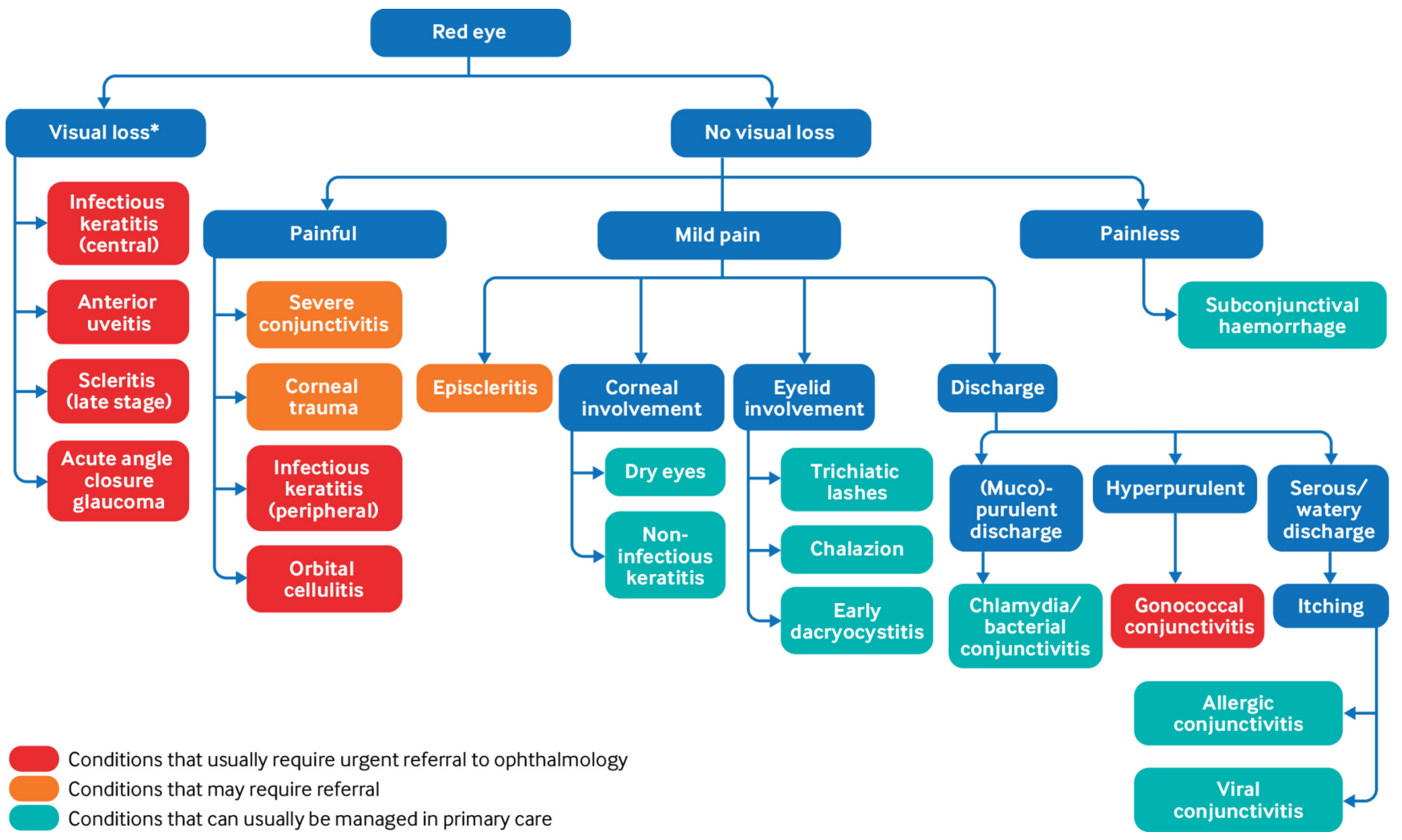

Fig 1 | Possible diagnoses based on initial assessment of red eye in primary care. *Red eye with visual loss is often caused by conditions that are painful and warrant an urgent ophthalmic examination. However, be aware that patients with neurotrophic keratopathy (reduced or absent corneal sensation) may not complain of any ocular pain despite having serious corneal pathology such as infectious keratitis

Virtual examination can be performed either via a live interactive video consultation, or a store-and-forward, asynchronous review of patient's self-taken images. ${ }^{3}$ Tailor the virtual examination according to the history.

Visual acuity-A crude estimation can be performed by asking the patient to read sentences of different font sizes (such as from a newspaper) at a fixed distance. Ask the patient to check one eye at a time, with the correct glasses (if any), as many patients are not aware of the loss of vision until they shut the unaffected eye. Comparing visual acuity between the eyes may provide additional information on level of visual impairment in the affected eye. In patients with diplopia (double vision), this helps to determine whether the diplopia is monocular (suggestive of ocular causes) or binocular (suggestive of ocular motility or neurological causes).

Free online mobile phone apps are available to check vision, but most of them have not gained regulatory approval. ${ }^{17}{ }^{18}$ In a prospective comparative study of app-based home vision testing in children, most families were able to generate results deemed useful for clinical decision making, but parental engagement was low. ${ }^{19}$

Face and eyelid-The presence of vesicles and rashes on the face or eyelids suggest herpes simplex infection or herpes zoster ophthalmicus (if the involvement respects the midline of the face and V1 dermatomal distribution (fig 2)). ${ }^{20}$ Presence of Hutchinson's sign (involvement of the tip or lateral aspect of the nose) suggests a higher likelihood of ocular involvement in herpes zoster ophthalmicus. ${ }^{20}$ Periorbital swelling may suggest preseptal (less serious) or orbital cellulitis (more serious), with the latter being associated with orbital involvement (for example, painful red eye, reduced visual acuity, proptosis, and restricted eye movement). Preseptal cellulitis can progress to orbital cellulitis, particularly in younger children where the orbital septum has not fully developed. Other aetiology such as angioedema, should be suspected when patient presents with periorbital swelling. Periocular eczema or inflammation is suggestive of allergic eye disease or contact dermatitis (when there is a recent history of using new eyedrops). 


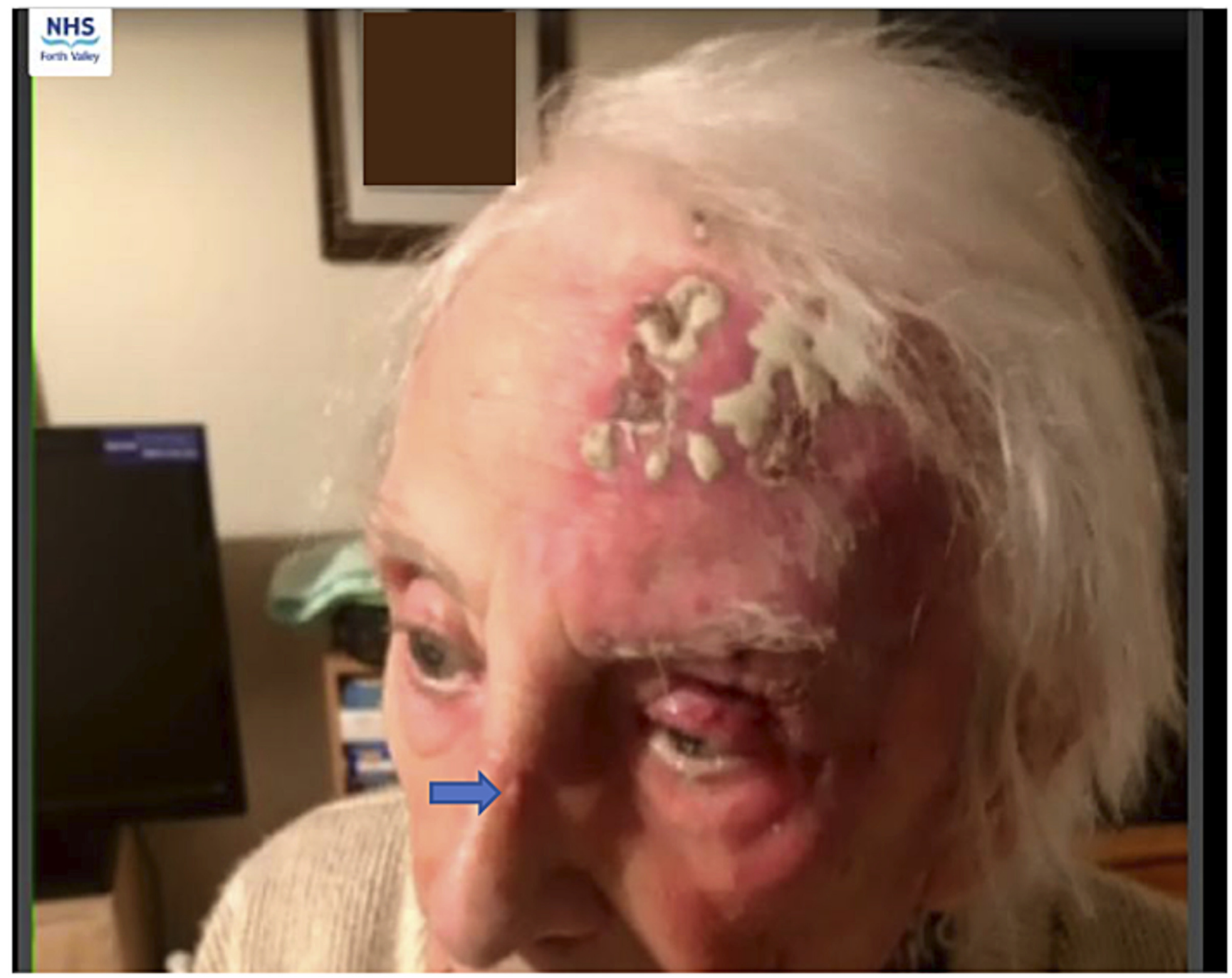

Fig 2 | A patient with left herpes zoster ophthalmicus. The screenshot taken from the video consultation from live NHS Near Me (powered by Attend Anywhere) demonstrates the presence of rashes and vesicles (respecting the midline of the face) with white-yellowish plaque-like changes is suggestive of herpes zoster ophthalmicus (positive Hutchinson's sign; blue arrow) with bacterial superinfection.

Conjunctiva-Look for localised or diffuse redness. Box 3 lists possible causes for conjunctival redness.

\section{Box 3: Differential diagnosis of conjunctival hyperaemia}

- Diffuse superficial hyperaemia-Conjunctivitis, dry eye, blepharitis, corneal infection (mild)

- Localised superficial hyperaemia-Subconjunctival haemorrhage, episcleritis

- Diffuse deep hyperaemia-Corneal infection (severe), acute angle-closure glaucoma, scleritis

- Circumcorneal hyperaemia-Anterior uveitis
Cornea-Ask the patient or family member to shine a light (such as a pen torch) from the corner of the eye without obstructing the camera, whilst looking straight ahead. The cornea is normally smooth and transparent. Any haziness or whitening may indicate corneal opacity, infiltrate, or corneal oedema. This can be secondary to corneal diseases, intraocular inflammation, or elevated intraocular pressure (fig 3). Corneal signs (unless severe) are not easily detectable on mobile technology. Refer patients with suspected corneal diseases, based on history, for a face-to-face examination. 


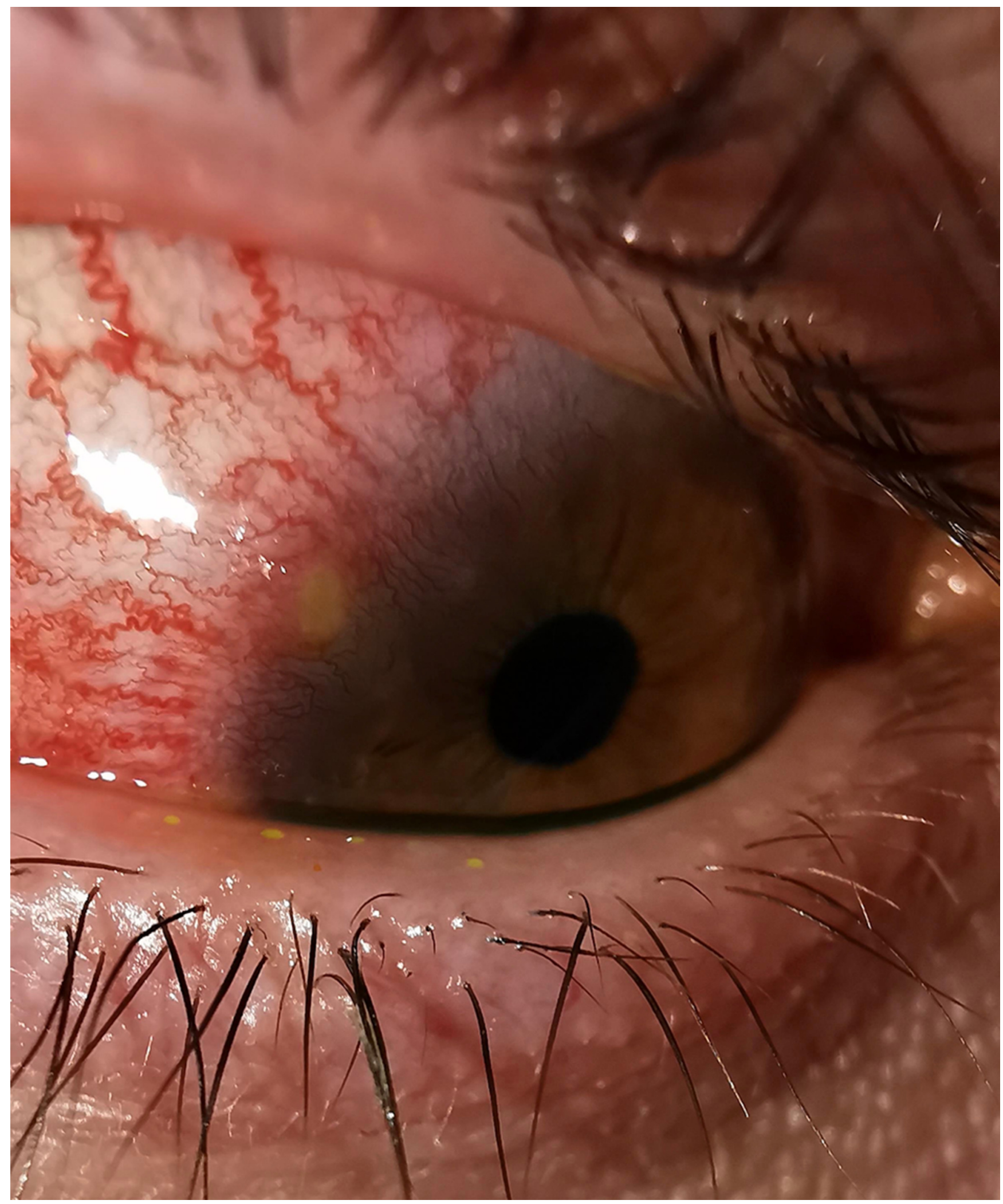

Fig 3 | A self taken photograph by a patient for a painful right red eye sent over a secure cloud-based platform. This patient was diagnosed with "keratitis," evidenced by the presence of a white infiltrate at the superotemporal limbus and marked conjunctival injection

Pupils-Inspect both pupils for size, shape, position, and symmetry by asking patient to bring the device close to their eyes. Any difference in size or symmetry of the pupils (anisocoria) warrants further ophthalmology assessment (fig 4 and video 1). ${ }^{21}$ Pupillary light responses, including direct and consensual light reflexes and relative afferent pupillary defect, should be checked when the vision 
is affected or orbital pathology is suspected. Examination can be performed by asking the patient to shine light into each eye in turn and swinging the light from one eye to another. A non-reactive pupil may be indicative of acute angle-closure glaucoma (tonic, mid-dilated pupil) or anterior uveitis (small, non-dilating pupil due to posterior synechiae). Relative afferent pupillary defect (RAPD) can be detected by moving the light between the two eyes quickly (spend about 3 seconds on each eye). Normally, both pupils should constrict when light is shone to either pupil. However, when RAPD is present in either eye, both pupils will dilate when light is shone to the abnormal eye (due to reduced afferent light impulse). This is indicative of significant disease at pre-chiasmal visual pathway, including retinal pathology or optic neuropathy.

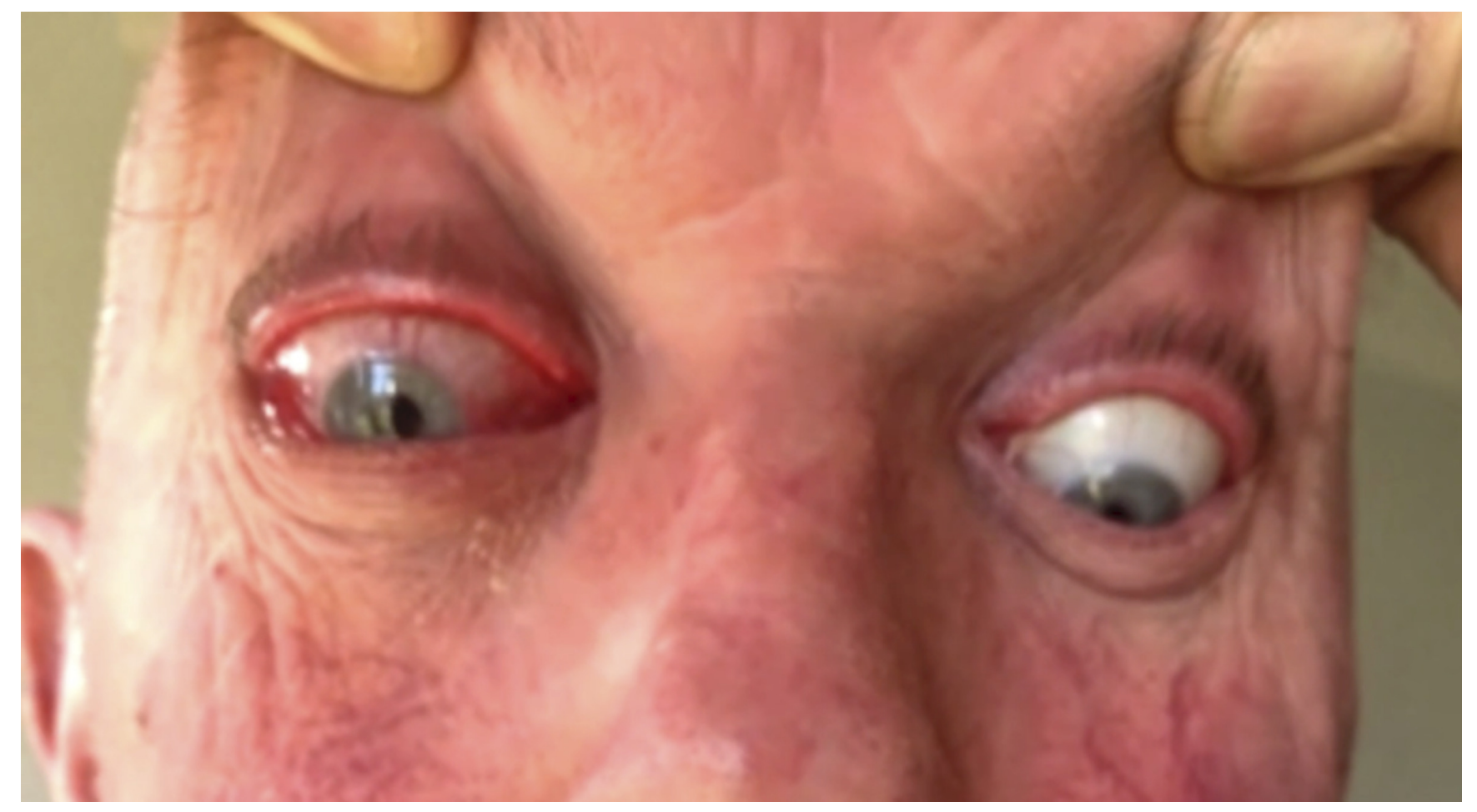

Fig 4 | A patient with a new onset of a right painful red eye with diplopia. Proptosis, restricted eye movement and anisocoria (right pupil is larger than the left pupil) was detected on a live video consultation (video 1). Visual acuity was checked by a family member using an app. An inter-eye acuity difference supported a diagnosis of right optic neuropathy. The patient was urgently referred for further management.

Video 1 Pupillary testing in a video consultation

Eyeball palpation-If there is no history of trauma to the eye, ask the patient to palpate the affected eyeball. Tenderness may suggest scleritis or acute angle-closure glaucoma. "Eyeball hardness" could be a sign of raised intraocular pressure, which warrants an urgent assessment by an ophthalmologist. However, this sign is very subjective and needs to be interpreted with caution.

\section{What you should do}

Recognising when to refer patients with a red eye to ophthalmology services, whether it is routine or urgently, is crucial (box 4). Figure 1 represents possible diagnoses to consider based on initial assessment.

\section{Box 4: Conditions that require urgent referral to an ophthalmologist}

- Severe "conjunctivitis" that does not improve after several days of antibiotic treatment ("bacterial conjunctivitis") or lubricants ("viral conjunctivitis")

- Corneal infection (especially in contact lens wearers)

- Anterior uveitis

- Acute angle-closure glaucoma

- Endophthalmitis (suspect if the patient had intra-ocular surgery within the past week)
- Ocular trauma (including mechanical, chemical, and thermal injury)

- Scleritis

- Orbital cellulitis

Patients with non-sight threatening red eye conditions-such as mild infectious or non-infectious conjunctivitis, subconjunctival haemorrhage, or mild corneal abrasion-can be reassured and managed in the community. ${ }^{22}$ Advise that most cases of acute viral and bacterial conjunctivitis will resolve without any treatment in 5-7 days. A trial of topical antibiotics (such as chloramphenicol or fusidic acid) can be started if symptoms are not resolving within three days of onset in bacterial conjunctivitis. ${ }^{23}$ If the condition does not improve or if it worsens after treatment, offer referral to an eye casualty unit for urgent ophthalmic assessment.

Suspected severe and potentially sight threatening conditions (box 4) or the presence of red flags (see box 1) warrant prompt ophthalmology referral. ${ }^{23}$ Patients with recurrent history of red eye related conditions warrant a face-to-face ophthalmology review. Ocular signs could be too subtle to be picked up by mobile imaging. Contact the local on-call ophthalmology team immediately for these patients. 


\section{Education into practice}

- Recollect a patient with an eye condition you examined recently. What would you do differently based on reading this article?

- What red flags would you look for in a patient with red eye that would prompt an ophthalmology referral?

\section{How patients were involved in the creation of this article}

Recent examples of patients with a red eye who had been assessed by virtual consultation, including telephone or video consultation, have informed the writing of this article. In addition, images of patients with red eyes taken during virtual consultations have been used after obtaining informed consent. We are grateful for their contribution.

\section{How this article was created}

We searched PubMed for relevant articles related to virtual consultation and red eye using several key words, including "red eye", "tele-medicine", "virtual consultation", "telephone consultation" and "video consultation". Literature search was last updated on 1 February 2021. We also sought expert opinions from ophthalmologists and general practitioners.

Contributors: CSH, AJA, and DSJT conceptualised and designed the study. CSH, IATL, and DSJT contributed to the data (and image) collection. All authors contributed to the data interpretation. CSH and DSJT drafted the manuscript. AJA and IATL critically revised the draft. All authors approved the final version of the manuscript. DSJT acts as the guarantor of this work.

Competing interests: We have read and understood BM/ policy on declaration of interests and have no relevant interests to declare.

Patient consent: Patient consent obtained.

Provenance and peer review: Commissioned, based on an idea from the author; externally peer reviewed.

1 Ting DSJ, Krause S, Said DG, Dua HS. Psychosocial impact of COVID-19 pandemic lockdown on people living with eye diseases in the UK. Eye (Lond) 2020;

doi: 10.1038/s41433-020-01130-4 pmid: 32778740

2 Kilduff CL, Thomas AA, Dugdill J, etal. Creating the Moorfields' virtual eye casualty: video consultations to provide emergency teleophthalmology care during and beyond the COVID-19 pandemic. BMJ Health Care Inform 2020;27:e100179. doi: 10.1136/bmihci-2020-100179 pmid: 32796085

3 Li JO, Liu H, Ting DSJ, etal. Digital technology, tele-medicine and artificial intelligence in ophthalmology: A global perspective. Prog Retin Eye Res 2020;100900.pmid: 32898686

4 Giardini ME, Livingstone IAT. Extending the reach and task-shifting ophthalmology diagnostics through remote visualisation. Adv Exp Med Biol 2020;1260:161-74. doi: 10.1007/978-3-030-47483-6_9 pmid: 33211312

5 Chen Y, Ismail R, Cheema MR, Ting DS,, Masri I. Implementation of a new telephone triage system in ophthalmology emergency department during COVID-19 pandemic: clinical effectiveness, safety and patient satisfaction. Eye (Lond) 2021;

doi: 10.1038/s41433-021-01528-8 pmid: 34035494

6 Wu X, Chen J, Yun D, etal. Effectiveness of an ophthalmic hospital-based virtual service during the COVID-19 pandemic. Ophthalmology 2021;128:942-5.

doi: 10.1016/j.ophtha.2020.10.012 pmid: 33069751

7 Napoli PE, Nioi M, d'Aloja E, Fossarello M. The ocular surface and the coronavirus disease 2019: Does a dual 'ocular route' exist?] Clin Med 2020;9:1269. doi: 10.3390/jcm9051269.

8 Napoli PE, Nioi M, d'Aloja E, Fossarello M. Safety recommendations and medical liability in ocular surgery during the COVID-19 pandemic: an unsolved dilemma. J Clin Med 2020;9:E1403. doi: 10.3390/jcm9051403 pmid: 32397530

9 Ting DSJ, Deshmukh R, Said DG, Dua HS. The impact of COVID-19 pandemic on ophthalmology services: are we ready for the aftermath? Ther Adv Ophthalmol 2020;12:2515841420964099. doi: 10.1177/2515841420964099 pmid: 33283153

10 Li JO, Thomas AAP, Kilduff CLS, etal. Safety of video-based telemedicine compared to in-person triage in emergency ophthalmology during COVID-19. EClinicalMedicine 2021;34:100818. doi: 10.1016/j.eclinm.2021.100818 pmid: 33842860

11 Frings A, Geerling G, Schargus M. Red eye: a guide for non-specialists. Dtsch Arztebl Int 2017;114:302-12. doi: 10.3238/arztebl.2017.0302. pmid: 28530180

12 Ting DSJ, Ho CS, Deshmukh R, Said DG, Dua HS. Infectious keratitis: an update on epidemiology, causative microorganisms, risk factors, and antimicrobial resistance. Eye (Lond) 2021;35:1084-101. doi: 10.1038/s41433-020-01339-3 pmid: 33414529

13 Guly CM, Forrester JV. Investigation and management of uveitis. BMJ 2010;341:C4976. doi: 10.1136/bmj.c4976 pmid: 20943722
14 Sun X, Dai Y, Chen Y, etal. Primary angle closure glaucoma: What we know and what we don't know. Prog Retin Eye Res 2017;57:26-45. doi: 10.1016/.jpreteyeres.2016.12.003 pmid: 28039061

15 Sims J. Scleritis: presentations, disease associations and management. Postgrad Med J 2012;88:713-8. doi: 10.1136/postgradmedj-2011-130282 pmid: 22977282

16 Azari AA, Barney NP. Conjunctivitis: a systematic review of diagnosis and treatment. JAMA 2013;310:1721-9. doi: 10.1001/jama.2013.280318 pmid: 24150468

17 Bastawrous A, Rono HK, Livingstone IA, etal. Development and validation of a smartphone-based visual acuity test (Peek Acuity) for clinical practice and community-based fieldwork. JAMA Ophthalmol 2015;133:930-7. doi: 10.1001/jamaophthalmol.2015.1468 pmid: 26022921

18 Kawamoto K, Stanojcic N, Li JO, Thomas PBM. Visual acuity apps for rapid integration in teleconsultation services in all resource settings: a review. Asia Pac J Ophthalmol (Phila) 2021. doi: 10.1097/AP0.0000000000000384 pmid: 33606386

19 Painter S, Ramm L, Wadlow L, O'Connor M, Sond B. Parental home vision testing of children during Covid-19 pandemic. Br Ir Orthopt J 2021;17:13-9doi: 10.22599/bioj.157.

20 Ting DSJ, Ghosh N, Ghosh S. Herpes zoster ophthalmicus. BMJ 2019;364:k5234. doi: 10.1136/bmj.k5234 pmid: 30655269

21 Rose GE, Pearson RV. Unequal pupil size in patients with unilateral red eye. BM/1991;302:571-2 doi: 10.1136/bmj.302.6776.571 pmid: 2021723

22 National Institute for Health and Care Excellence. Clinical Knowledge Summaries. Red eye: Management of red eye. 2016. https://cks.nice.org.uk/topics/red-eye/management/managementof-red-eye/.

23 National Institute for Health and Care Excellence. Clinical Knowledge Summaries. Conjunctivitis - infective: Management in primary care. 2021. https://cks.nice.org.uk/topics/conjunctivitis-infec tive/management/management-in-primary-care/.

This is an Open Access article distributed in accordance with the Creative Commons Attribution Non Commercial (CC BY-NC 4.0) license, which permits others to distribute, remix, adapt, build upon this work non-commercially, and license their derivative works on different terms, provided the original work is properly cited and the use is non-commercial. See: http://creativecommons.org/licenses/by$n c / 4.0 /$. 